\title{
Vulnerabilidade social e incidência de COVID-19 em uma metrópole brasileira
}

\author{
Social vulnerability and COVID-19 incidence \\ in a Brazilian metropolis
}

\author{
Virna Ribeiro Feitosa Cestari (http://orcid.org/0000-0002-7955-0894) ${ }^{1}$ \\ Raquel Sampaio Florêncio (https://orcid.org/0000-0003-3119-7187) ${ }^{1}$ \\ George Jó Bezerra Sousa (https://orcid.org/0000-0003-0291-6613) ${ }^{1}$ \\ Thiago Santos Garces (https://orcid.org/0000-0002-1670-725X) ${ }^{1}$ \\ Thatiana Araújo Maranhão (https://orcid.org/0000-0003-4003-1365) ${ }^{2}$ \\ Révia Ribeiro Castro (https://orcid.org/0000-0002-9260-4148) ${ }^{1}$ \\ Luana Ibiapina Cordeiro (https://orcid.org/0000-0002-3128-6000) ${ }^{1}$ \\ Lara Lídia Ventura Damasceno (https://orcid.org/0000-0002-0496-5622) ${ }^{1}$ \\ Vera Lucia Mendes de Paula Pessoa (https://orcid.org/0000-0002-8158-7071) ${ }^{1}$ \\ Maria Lúcia Duarte Pereira (http://orcid.org/0000-0002-7685-6169) ${ }^{1}$ \\ Thereza Maria Magalhães Moreira (https://orcid.org/0000-0003-1424-0649) ${ }^{1}$
}

${ }^{1}$ Universidade Estadual do Ceará. Av. Dr. Silas Muguba 1700, Itaperi. 60714-903 Fortaleza CE Brasil. virna.ribeiro@hotmail.com

${ }^{2}$ Departamento de

Enfermagem, Universidade

Estadual do Piauí. Parnaíba PI Brasil.

\begin{abstract}
Vulnerability is a crucial factor in addressing COVID-19 as it can aggravate the disease. Thus, it should be considered in COVID-19 control and health prevention and promotion. This ecological study aimed to analyze the spatial distribution of the incidence of COVID-19 cases in a Brazilian metropolis and its association with social vulnerability indicators. Spatial scan analysis was used to identify COVID-19 clusters. The variables for identifying the vulnerability were inserted in a Geographically Weighted Regression (GWR) model to identify their spatial relationship with COVID-19 cases. The incidence of COVID-19 in Fortaleza was 74.52/10,000 inhabitants, with 3,554 reported cases and at least one case registered in each neighborhood. The spatial GWR showed a negative relationship between the incidence of COVID-19 and demographic density $(\beta=-0,0002)$ and a positive relationship between the incidence of COVID-19 and the percentage of self-employed $>18$ years $(\beta=1.40)$, and maximum per capita household income of the poorest fifth $(\beta=0.04)$. The influence of vulnerability indicators on incidence showed areas that can be the target of public policies to impact the incidence of COVID-19.
\end{abstract}

Key words Coronavirus, Social Vulnerability, Ecological Studies
Resumo A vulnerabilidade é um fator chave no enfrentamento da COVID-19 tendo em vista que pode influenciar no agravamento da doença. Desse modo, ela deve ser considerada no controle da COVID-19, prevenção e promoção da saúde. O objetivo deste artigo é analisar a distribuição espacial da incidência de casos de COVID-19 em uma metrópole brasileira e sua associação com indicadores de vulnerabilidade social. Estudo ecológico. Foi utilizada a análise de varredura espacial (scan) para identificar aglomerados de COVID-19. As variáveis para identificação da vulnerabilidade foram inseridas em um modelo de Regressão Espacial Geograficamente Ponderado (GWR) para identificar sua relação espacial com os casos de COVID-19. A incidência de COVID-19 em Fortaleza foi de 74,52/10 mil habitantes, com notificação de 3.554 casos, sendo pelo menos um caso registrado em cada bairro. A regressão espacial GWR mostrou relação negativa entre incidência de COVID-19e densidade demográfica $(\beta=-0,0002)$ e relação positiva entre incidência de COVID-19 e percentual de ocupados $>18$ anos trabalhadores autônomos $(\beta=1,40)$, assim como, renda domiciliar per capita máxima do quinto mais pobre $(\beta=0,04)$. A influência dos indicadores de vulnerabilidade sobre a incidência evidenciou áreas que podem ser alvo de políticas públicas a fim de impactar na incidência de COVID-19.

Palavras-chave Coronavírus, Vulnerabilidade Social, Estudos Ecológicos 


\section{Introdução}

A Coronavirus Disease 2019 (COVID-19) possui como agente etiológico o novo beta coronavírus 2, causador da Síndrome Respiratória Aguda Grave (SARS-CoV-2). Em 2020, foi declarada pela Organização Mundial de Saúde (OMS) uma Emergência de Saúde Pública de Importância Internacional, portanto, pandêmica, com elevada transmissibilidade e rápida letalidade em todos os continentes ${ }^{1}$.

Mundialmente, desde o primeiro caso revelado em Wuhan, China, no final de 2019, até 15 de junho de 2020 foram confirmados 7.283.289 casos e 431.541 óbitos. No mesmo período, a América ocupava o primeiro lugar no ranking, com 3.841.609 casos e 203.574 óbitos². Nesse ínterim, os países americanos com mais casos até 13 de agosto de 2020 são: Estados Unidos (América do Norte) com 5.217.094 casos e Brasil (América do Sul) com 3.180 .758 casos $^{3}$.

No Brasil, desde o início da disseminação da doença o Ministério da Saúde atuou junto ao Centro de Operações de Emergência (COE) no planejamento, organização e monitoramento de ações nesse cenário epidemiológico. Entre os estados brasileiros mais afetados estão São Paulo e Rio de Janeiro no Sudeste brasileiro, e o Ceará, no Nordeste. Este último, por sua vez, confirmou 195.298 mil casos até o dia 13 de agosto de $2020^{4}$. Dentre esses, 66,3\% são residentes em Fortaleza, a capital do Estado 5,6 .

Com população de 2,7 milhões, Fortaleza teve os primeiros casos registrados de COVID-19 do Estado em março de 2020, localizados nos bairros mais ricos e com melhor Índice de Desenvolvimento Humano (IDH). O vírus entrou na cidade por meio de moradores infectados em viagens ao exterior e, atualmente, se propaga pela zona periférica, detentora da população mais pobre ${ }^{7,8}$.

Além do agravante epidemiológico, a Capital Fortaleza tem um contexto de desigualdade social marcante quanto às condições de habitação, renda e estrutura demográfica ${ }^{9}$, a qual impõe ao poder público à necessidade de vigilância premente para identificar espaços de maior vulnerabilidade social, com vistas à otimização do controle da dispersão e prevenção da COVID-19. Nesse sentido, estudos apontam para o acometimento de grupos populacionais com maio vulnerabilidade, em função de suas condições de vida e situação de saúde ${ }^{10-12}$.

Nesse sentido, sabe-se que o contexto socioeconômico é decisivo na maior vulnerabilidade à doença, funcionando como um propulsor para expansão do novo coronavírus ${ }^{13,14}$. Assim, a população em vulnerabilidade social é a mais impactada pelos seus efeitos, dada a ausência e/ ou insuficiência de recursos, estratégias de prevenção e/ou tratamento da doença em seus cotidianos, associados às dificuldades de realizar o isolamento social, manutenção do emprego e renda, bem como menor acesso à saúde e saneamento básico ${ }^{15-17}$. Desse modo, esse cenário de vulnerabilidade social deve ser considerado nas ações de promoção da saúde, prevenção e controle da COVID-19.

Neste estudo, considerou-se a vulnerabilidade social como uma condição de precariedade produzida pelas diferentes e desiguais formas de o sujeito interagir com outras vidas ou instituições no campo da saúde, sendo referente à situação socioeconômica, identidade demográfica, cultura, contexto familiar, redes e suporte socias, gênero, violência, controle social e ecossistema ${ }^{18}$. Tal perspectiva traz uma compreensão mais alargada das ações da política de saúde sobre os múltiplos fatores que incidem no cotidiano de vida dos sujeitos em seus territórios ${ }^{19}$.

Baseado nisso, o objetivo deste estudo foi analisar a distribuição espacial da incidência de casos de COVID-19 nesta metrópole brasileira e sua associação com indicadores de vulnerabilidade social. O estudo em questão analisou dados relativos aos meses de março e abril que contivessem a localização geográfica do caso, e tivessem sido disponibilizados pelo Governo do Estado do Ceará em base dados públicos.

\section{Método}

Estudo ecológico, cujas unidades de análise foram os bairros de Fortaleza. Segundo o Instituto Brasileiro de Geografia e Estatística (IBGE), a cidade tem 121 bairros, 2.669.342 habitantes e $312,353 \mathrm{~km}^{2}$ de área, com densidade demográfica de $7.86,44 \mathrm{hab} / \mathrm{Km}^{2}$ com base nos dados dos Censos Demográficos de 1991, 2000 e 2010. É a cidade mais populosa do estado e a quinta mais populosa do Brasil. Possui o décimo maior produto interno bruto do país, acumulando riquezas e também desigualdades, pois sua renda é concentrada em poucos bairros, de elevado IDH, embora a maioria de seus bairros tenha IDH menor que 0,5 , considerado muito baixo (Figura 1 ).

Ademais, os dados desta investigação são do tipo secundário, sendo utilizada como fonte destes o sítio eletrônico do IntegraSUS, que abriga informações referentes ao número de casos e 

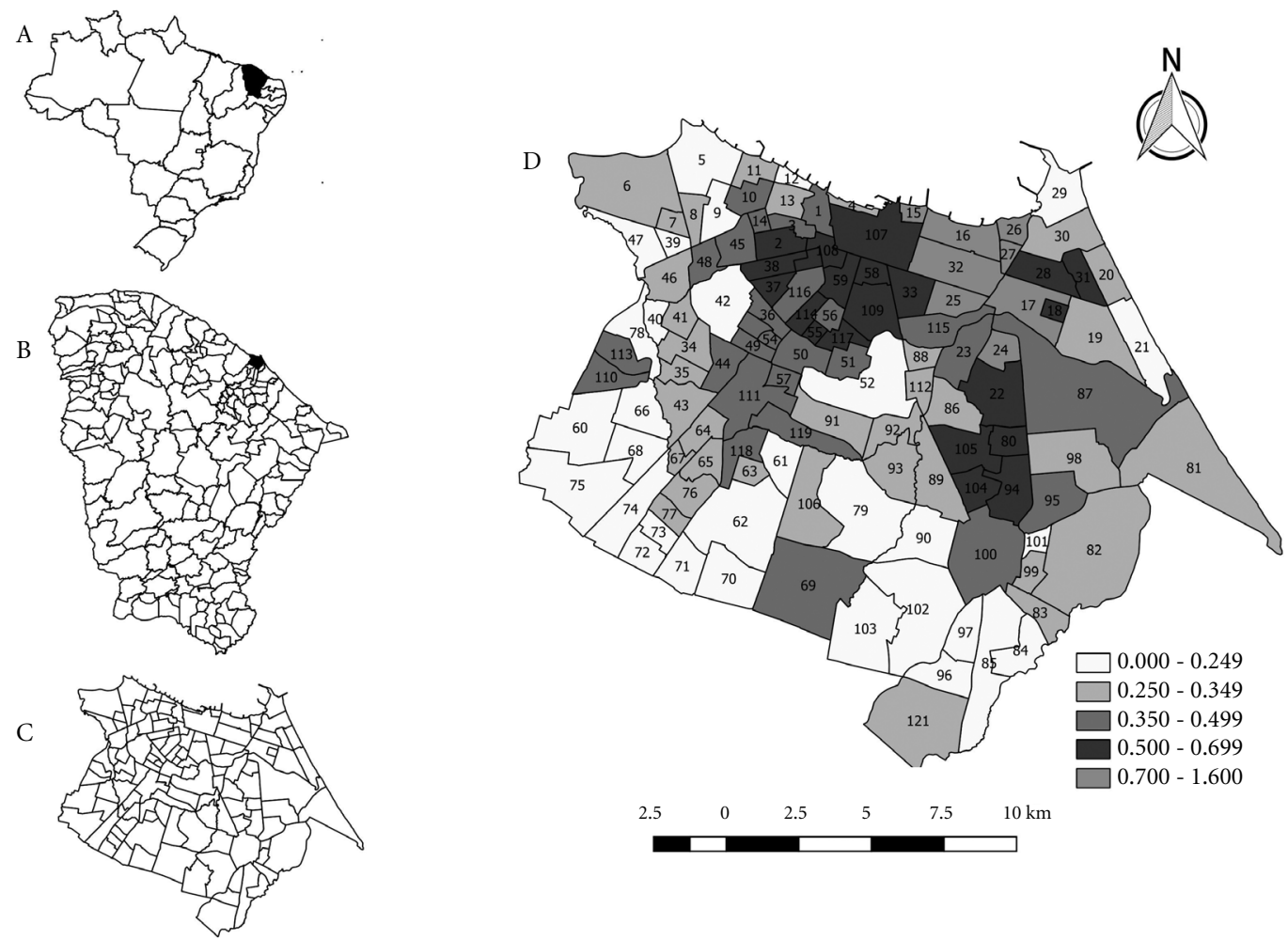

Figura 1. Figura 1A. Localização do Estado do Ceará no Brasil. Figura 1B: Localização de Fortaleza no Estado do Ceará. Figura 1C: Mapa de Fortaleza. Figura 1D: Mapa de Fortaleza com o IDH de seus bairros.

Códigos: 1 Jacarecanga; 2 São Gerardo; 3 Monte Castelo; 4 Moura Brasil; 5 Barra do Ceará; 6 Vila Velha; 7 Jardim Guanabara; 8 Jardim Iracema; 9 Floresta; 10 Álvaro Weyne; 11 Cristo Redentor; 12 Pirambu; 13 Carlito Pamplona; 14 Ellery; 15 Praia de Iracema; 16 Meireles; 17 Cocó; 18 Cidade 2000; 19 Manuel Dias Branco; 20 Praia do Futuro I; 21 Praia do Futuro II; 22 Engenheiro Luciano Cavalcante; 23 Salinas; 24 Guararapes; 25 Dionísio Torres; 26 Mucuripe; 27 Varjota; 28 Papicu; 29 Cais do Porto; 30 Vicente Pinzón; 31 De Lourdes; 32 Aldeota; 33 Joaquim Távora; 34 Henrique Jorge; 35 João XXIII; 36 Bela Vista; 37 Amadeu Furtado; 38 Parquelândia; 39 Olavo Oliveira; 40 Autran Nunes; 41 Dom Lustosa; 42 Pici; 43 Bonsucesso; 44 Jóquei Clube; 45 Presidente Kennedy; 46 Antônio Bezerra; 47 Quintino Cunha; 48 Padre Andrade; 49 Demócrito Rocha; 50 Montese; 51 Vila União; 52 Aeroporto; 53 Panamericano; 54 Couto Fernandes; 55 Bom Futuro; 56 Jardim América; 57 Itaoca; 58 José Bonifácio; 59 Benfica; 60 Granja Lisboa; 61 Dendê; 62 Mondubim; 63 Jardim Cearense; 64 Vila Peri; 65 Manoel Sátiro; 66 Granja Portugal; 67 Parque São José; 68 Bom Jardim; 69 Prefeito José Walter; 70 Planalto Ayrton Senna; 71 Aracapé; 72 Parque Presidente Vargas; 73 Parque Santa Rosa; 74 Canindezinho; 75 Siqueira; 76 Novo Mondubim; 77 Conjunto Esperança; 78 Genibaú; 79 Passaré; 80 Parque Manibura; 81 Sabiaguaba; 82 Lagoa Redonda; 83 Coaçu; 84 São Bento; 85 Paupina; 86 Jardim das Oliveiras; 87 Edson Queiroz; 88 Alto da Balança; 89 Cajazeiras; 90 Barroso; 91 Serrinha; 92 Dias Macêdo; 93 Boa Vista/Castelão; 94 Cambeba; 95 José de Alencar; 96 Ancuri; 97 Parque Santa Maria; 98 Sapiranga/Coité; 99 Guajeru; 100 Messejana; 101 Curió; 102 Jangurussu; 103 Conjunto Palmeiras; 104 Parque Iracema; 105 Cidade dos Funcionários; 106 Parque Dois Irmãos; 107 Centro; 108 Farias Brito; 109 Fátima; 110 Conjunto Ceará II; 111 Parangaba; 112 Aerolândia; 113 Conjunto Ceará I; 114 Damas; 115 Tauape; 116 Rodolfo Teófilo; 117 Parreão; 118 Maraponga; 119 Itaperi; 120 Parque Araxá; 121 Pedras.

Fonte: Elaborado pelo autor.

indicadores da COVID-19 no Ceará disponíveis em domínio público ${ }^{20}$. Os dados analisados são referentes aos meses de março e abril. A coleta de dados ocorreu em maio de 2020 e contemplou somente os casos que continham o bairro de ocorrência em sua notificação. Vale ressaltar que a base de dados estadual contém todos os casos testados para a doença desde o primeiro caso suspeito. O critério de inclusão foi a disponibilidade da informação de localização geográfica do caso. 
Em relação as variáveis associadas ao desfecho, o Atlas de Desenvolvimento Humano no Brasil aborda mais de 200 indicadores de vulnerabilidade social nas áreas de demografia, educação, renda, trabalho e habitação, com dados dos Censos Demográficos de 1991, 2000 e 2010. Para análise foram considerados os indicadores: densidade demográfica, analfabetismo, ensino fundamental e médio na população $>18$ anos de idade, percentual da população de $>25$ anos com curso superior completo, índice de Gini, renda per capita média, índice de Theil-L, percentual (\%) de ocupados $>18$ anos trabalhadores por conta própria, empregados com carteira e sem ocupação, \% da população com água encanada, com banheiro e água encanada, que vive em domicílios com $>2$ pessoas por dormitório, $\% \mathrm{da}$ população que vive em domicílios urbanos com serviço de coleta de lixo, com abastecimento de água e esgotamento sanitário inadequados, \% de pessoas em domicílios com paredes que não são de alvenaria ou madeira aparelhada, $\%$ de pessoas em domicílios vulneráveis à pobreza e que gastam mais de uma hora até o trabalho no total de pessoas ocupadas, $\%$ de pessoas em domicílios sem energia elétrica, \% de pessoas vulneráveis e dependentes de idosos, no total de pessoas em domicílios vulneráveis e com idosos, população de mulheres chefes de família com, pelo menos, um filho $<15$ anos de idade, população em domicílios vulneráveis e com idoso, população ocupada vulnerável à pobreza que retorna diariamente do trabalho para o domicílio, Índice de Desenvolvimento Humano e renda domiciliar per capita máxima do quinto mais pobre. Todos esses indicadores foram retirados do censo de 2010.

Inicialmente, realizou-se análise descritiva dos dados, contemplando a frequência simples e relativa ou mediana e intervalo interquartílico (IQR), visando descrever o perfil epidemiológico dos casos de COVID-19 em Fortaleza. A partir disso, calculou-se a incidência da doença na cidade, utilizando o número de casos acumulados em cada bairro no numerador e a população dos bairros no ano 2010 como denominador, multiplicado por 10.000 habitantes. A constante 10.000 foi selecionada para favorecer a comparação dos bairros, visto que alguns são populosos e outros não.

Para a análise espacial, foi criado o mapa temático da incidência de COVID-19 em cada bairro e suavizadas as taxas via método bayesiano para diminuir a instabilidade causada pelas diferenças entre estes. Esse método considera o valor do bairro, mas o pondera em relação aos bairros de fronteira por uma matriz de proximidade espacial, considerando o critério de contiguidade, no qual se atribui valor 1 àqueles com margens em comum e 0 aos que não as compartilham.

Em seguida, realizou-se varredura espacial scan, empregada para identificar clusters, bem como áreas de risco para COVID-19. Para tanto, calculou-se o Risco Relativo (RR) de cada bairro para a incidência da doença e identificou-se a presença de aglomerados espaciais. Para identificar os aglomerados espaciais utilizou-se o modelo discreto de Poisson e os requisitos foram: não sobreposição geográfica dos aglomerados, tamanho máximo do aglomerado igual a $50 \%$ da população exposta, aglomerados em formato circular e 999 replicações.

Por fim, para identificar fatores relacionados à incidência da doença, os indicadores foram inseridos em um modelo de regressão não espacial Ordinary Least Square (OLS) step forward com valor de entrada de 0,1. Aqueles que permaneceram no modelo final foram também inseridos em um modelo de Regressão Espacial Geograficamente Ponderado (GWR), pois, além de utilizar valores dos indicadores de determinado bairro, ele também considera valores dos bairros vizinhos, utilizando-se uma matriz de proximidade espacial pelo critério de contiguidade. Por fim, o resultado da regressão GWR foi apresentado em mapas temáticos

O cálculo da taxa bayesiana empírica local foi feito no software TerraView 4.2.2, a análise de varredura puramente espacial foi realizada no software SaTScan 9.6. já a regressão não espacial OLS foi realizada no software Stata 12 e a regressão espacial GWR no software GWR4.0.9. Todos os mapas foram produzidos no software QGIS 2.4.17.

Este estudo não necessitou de aprovação prévia de Comitê de Ética em Pesquisa, dado que o banco de dados da COVID-19 e o Atlas de Desenvolvimento Humano do Brasil são informações de domínio público, disponibilizados nos sítios eletrônicos do Governo do Estado do Ceará e do Instituto de Pesquisa Econômica Aplicada (IPEA), respectivamente. Ressalta-se ainda a impossibilidade na identificação do paciente, pois informações como nome ou endereço não foram disponibilizadas. Reitera-se o compromisso ético dos pesquisadores adotado no manuseio, análise e publicação dos dados, conforme preconiza a Resolução 510 de 2016 do Conselho Nacional de Pesquisa. 
Até o dia 28 de abril de 2020, Fortaleza notificou 3.554 casos de COVID-19. Os pacientes possuíam idade mediana de 47 anos (IQR:35-61), sendo a maioria do sexo feminino $(52,9 \% ; n=1.880)$. Junto a isso, as comorbidades mais comumente notificadas foram as doenças cardiovasculares $(6,5 \% ; n=232)$ e diabetes $(4,9 \% ; n=175)$. Do total de casos, $14,2 \%(n=505)$ foram internados, sendo 3,4\% $(n=122)$ destes em Unidade de Tratamento Intensivo (UTI) (Tabela 1).

Sobre a distribuição espacial da COVID-19, a Figura 2A evidencia que todos os bairros de Fortaleza registraram, pelo menos, um caso da doença e alguns bairros acumulam incidência bruta de até 74,52/10.000 habitantes, sendo estes localizados na periferia da cidade (Pedras e Mondubim). Quando suavizados, identificou-se que os bairros mais ricos (Meireles, Aldeota, Mucuripe, Papicu e Cocó) ainda possuíam relevante papel na incidência da doença, pois concentraram os casos durante semanas (Figura 2B).

Pela varredura scan, foi possível identificar que o risco de adoecimento por COVID-19 na cidade variou até 5,26 vezes em bairros periféricos (Pedras e Mondubim) e que bairros ricos, como Meireles e Aldeota, possuem risco 2 a 4 vezes maior que o restante do município (Figura 2). Também foram identificados oito aglomera- dos estatisticamente significantes para a doença na cidade (Figura 2D). O cluster mais provável, círculo menor, tem seis bairros (Meireles, Aldeota Varjota, Mucuripe, Papicu e Cocó) e RR 3,06 vezes maior de adoecimento, se comparado aos demais bairros $(\mathrm{p}<0.001)$.

A Tabela 2 apresenta o modelo final da regressão OLS, que demonstrou a influência de indicadores de vulnerabilidade sobre a incidência da COVID-19 que, por sua vez, apontou quatro variáveis estatisticamente significativas para o desfecho: renda domiciliar per capita máxima do quinto mais pobre $(\mathrm{p}<0,001)$; percentual de ocupados $\geq 18$ anos ou mais, que são trabalhadores por conta própria $(\mathrm{p}=0,03)$; percentual da população de $\geq 18$ anos com fundamental completo $(\mathrm{p}=0,04)$; e densidade demográfica $(\mathrm{p}=0,04)$.

Quando estas variáveis foram inseridas no modelo GWR, foi possível identificar a influência delas no espaço. O modelo mostrou relação negativa entre incidência de COVID-19 e população $\geq 18$ anos com fundamental completo $(\beta=-$ $0,26)$ e densidade demográfica $(\beta=-0,0002)$. Por outro lado, observou-se relação positiva entre incidência de COVID-19 e percentual de ocupados $\geq 18$ anos trabalhadores autônomos $(\beta=1,40)$, assim como, renda domiciliar per capita máxima do quinto mais pobre $(\beta=0,04)$ (Tabela 2 ). Ressalta-se que densidade demográfica e renda domiciliar per capta máxima do quinto mais po-

Tabela 1. Caracterização dos casos notificados de COVID-19 em Fortaleza.

\begin{tabular}{lcc}
\hline \multicolumn{1}{c}{ Variáveis } & $\mathbf{n}(\%)$ & $\mathbf{n}(\%)$ \\
\hline Idade (mediana/intervalo interquartílico) & Feminino & \\
Sexo & $1880(52,9)$ & Masculino \\
& Sim & $1674(47,1)$ \\
Doenças e internação & $12(0,4)$ & Não \\
Asma & $232(6,5)$ & $3542(99,6)$ \\
Doenças cardiovasculares & $175(5,0)$ & $3322(93,5)$ \\
Diabetes & $12(0,3)$ & $3379(95,0)$ \\
Imunodeficiências & $28(0,8)$ & $3542(99,7)$ \\
Doenças neurológicas & $9(0,2)$ & $3526(99,2)$ \\
Obesidade & $27(0,8)$ & $3545(99,8)$ \\
Pneumopatias & $29(0,8)$ & $3527(99,2)$ \\
Doenças renais & $505(14,2)$ & $3525(99,2)$ \\
Internação & $122(3,4)$ & $3049(85,8)$ \\
Admissão em UTI & & $3554(100,0)$ \\
Total & & \\
\hline
\end{tabular}

$\mathrm{n}$ = Número da Amostra; \%: Porcentagem.

Fonte: Elaborado pelo autor. 
bre possuem coeficientes muito próximos a zero, devendo assim serem interpretados com cautela.

Ademais, os mapas temáticos dos resultados podem ser observados na Figura 3, exceto a associação entre incidência da COVID-19 e porcentagem da população $\geq 18$ anos com fundamental completo, pois o modelo GWR não identificou relação estatisticamente significativa em nenhum bairro.
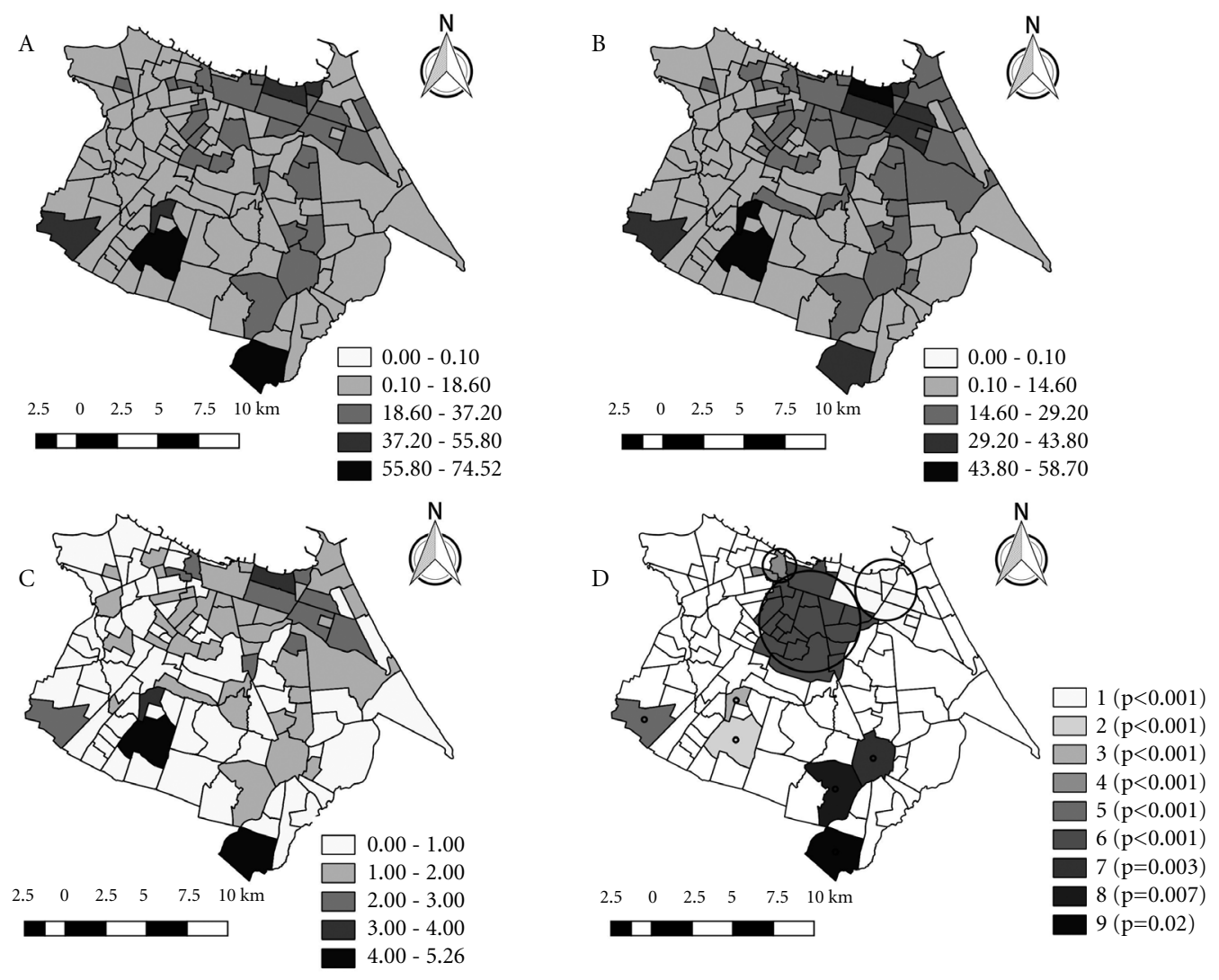

Figura 2. Incidência bruta e Bayesiana da COVID-19 em Fortaleza, risco de adoecimento e prováveis clusters de infecção.

Fonte: Elaborado pelo autor.

Tabela 2. Modelo final da regressão OLS step forward e GWR para incidência de COVID-19.

\begin{tabular}{|c|c|c|c|c|c|}
\hline \multirow{2}{*}{$\begin{array}{c}\text { Variáveis } \\
\text { Indicadores de vulnerabilidade social }\end{array}$} & \multicolumn{2}{|c|}{ Regressão OLS } & \multirow[b]{2}{*}{ p-valor } & \multicolumn{2}{|c|}{ Regressão GWR } \\
\hline & Coeficiente & Erro Padrão & & Coeficiente & Erro Padrão \\
\hline $\begin{array}{l}\text { Renda domiciliar per capita máxima } \\
\text { do quinto mais pobre }\end{array}$ &, 042 &, 010 & $<0,001$ & 0,04 & 0,002 \\
\hline $\begin{array}{l}\% \text { de ocupados } \geq 18 \text { anos trabalhadores } \\
\text { autônomos }\end{array}$ & 1,36 & ,628 & 0,03 & 1,40 & 0,18 \\
\hline $\begin{array}{l}\% \text { da população } \geq 18 \text { anos com } \\
\text { fundamental completo }\end{array}$ &,- 323 & ,16 & 0,04 & $-0,26$ & 0,04 \\
\hline Densidade demográfica &,- 0002 &, 0001 & 0,04 & $-0,0002$ & 0,0002 \\
\hline Constante & $-1,22$ & 16,15 & & $-5,70$ & 3,76 \\
\hline
\end{tabular}

Regressão OLS: $\mathrm{R}^{2}=0,2399 ; \mathrm{R}^{2}$ ajustado $=0,2137$; Regressão GWR: $\mathrm{R}^{2}=0,2816 ; \mathrm{R}^{2}$ ajustado $=0,2165$.

Fonte: Elaborado pelo autor. 


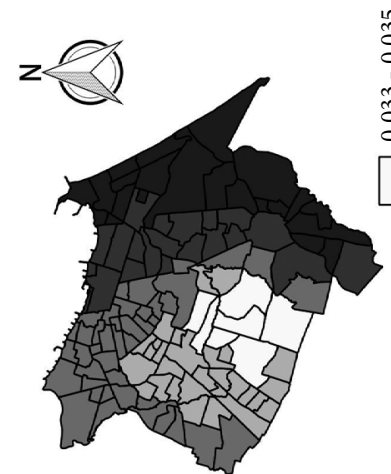

띠

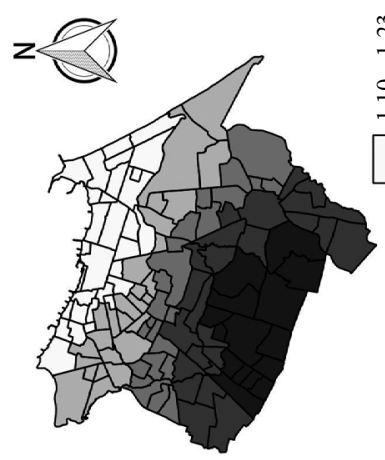

$\cup$

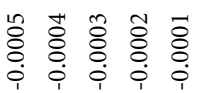

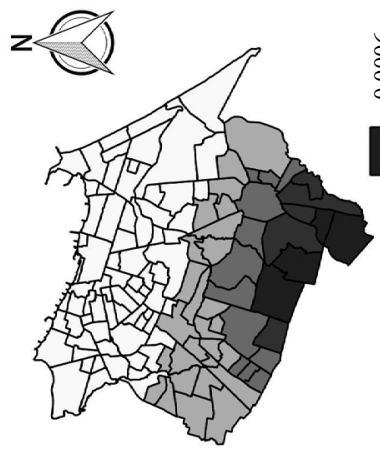

$\varangle$

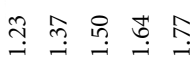

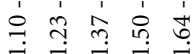
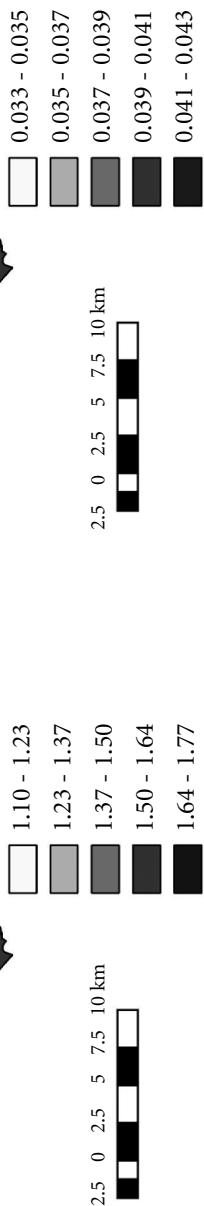

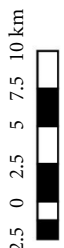

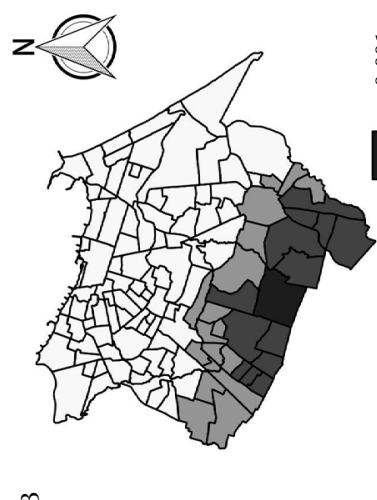

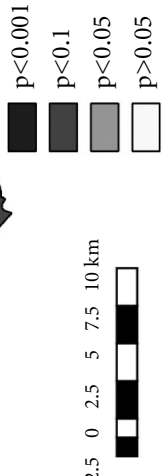

Figura 3. Coeficientes e p-valores da regressão GWR para indicadores de vulnerabilidade à COVID-19 em Fortaleza.

Nota: Figura 3A: Valor $\beta$ para densidade demográfica. Figura 3B: p-valor da densidade demográfica. Figura 3C: Valor $\beta$ para $\%$ de ocupados $>18$ anos trabalhadores autônomos. Figura 3D: p-valor \% de ocupados $>18$ anos trabalhadores autônomos. Figura 3E: Valor $\beta$ para renda domiciliar per capita máxima do quinto mais pobre. Figura 3F: p-valor da renda domiciliar per capita máxima do quinto mais pobre. 


\section{Discussão}

A pandemia de COVID-19 requer reorganização das sociedades contemporâneas e tem grande impacto, principalmente, em países e regiões com maiores desigualdades sociais e econômicas. Nessa perspectiva, o enfrentamento da pandemia perpassa o campo biológico e setores da saúde, repercutindo na economia, política e sociedade, o que demonstra a necessidade de atenção às condições que aumentam a vulnerabilidade em saúde da população.

Em todo o mundo, a disseminação do vírus é expressiva nas periferias e é certo que essa parcela da população sofra demasiadamente pela alta densidade de habitantes por casa, uso de transportes coletivos e fragilização do vínculo empregatício. Essas situações, por sua vez, favorecem a vulnerabilidade em saúde, condição humana caracterizada pela interação sujeito-social, sendo que essa relação produz precariedade quando o agenciamento não é tecido pelo sujeito ou coletivo no contexto da saúde ${ }^{18}$. Ressalta-se que neste estudo utilizou-se a densidade demográfica de todo o bairro ao invés da habitacional por serem os únicos disponíveis pelo IBGE.

É possível observar ainda que as cidades com números elevados da COVID-19 estão entre as mais populosas, como Nova York, nos Estados Unidos (EUA) ${ }^{21}$ e Mumbai, na Índia ${ }^{22}$. O surto do Coronavírus em Nova York iniciou em março de 2020, atingindo 100 casos em 5 dias e 10.000 casos em 22 de março ${ }^{21}$.

No contexto nacional, o Brasil comporta cidades populosas que atingiram elevados números de casos, como São Paulo, Rio de Janeiro e Fortaleza. Esta última, apresentou altos índices de casos de rápido crescimento e com dois picos em maio ${ }^{23}$. Vinculada ao turismo e a viagens, essa epidemia se caracteriza, inicialmente, por disseminação entres as classes média e alta, nas quais verificaram-se grande quantidade de casos e incidências em regiões geográficas mais abastadas das grandes capitais.

Embora muitas pesquisas estejam em desenvolvimento, a relação causal entre a COVID-19 e o território ainda não foi totalmente estabelecida. Entretanto, algumas inferências podem ser realizadas, visto que na presente pesquisa o perfil das pessoas com COVID-19 é semelhante ao de outros estudos realizados no estado ${ }^{7,8}$ e nas demais regiões do Brasil ${ }^{9}$.

Neste estudo, Fortaleza, a cidade mais populosa do estado, com território de maioria urbana e alta densidade demográfica, apresentou incidência elevada da COVID-19, semelhante à de Mumbai, cidade no Oeste da Îndia, com alta densidade populacional urbana e que representa $20 \%$ dos casos daquele país ${ }^{22}$, bem como com outras áreas urbanas de maior intensidade epidêmica e densidade populacional alta ${ }^{24}$.

Outrossim, a distribuição espacial revelou-se heterogênea, com desproporcional distribuição da doença entre bairros ricos e periféricos. A incidência foi maior nos bairros localizados na região Norte, com menor densidade demográfica, e foi menor em bairros localizados no extremo Sul do município, com alta densidade demográfica. Um estudo de análise espacial da COVID-19 realizado nas 24 regiões administrativas de Mumbai apresentou distribuição heterogênea distinta, com número de casos menor em regiões menos povoadas e número de casos maiores em regiões mais populosas ${ }^{22}$.

A discrepância entre Fortaleza e Mumbai pode estar relacionada aos aspectos socioeconômicos, visto que a zona Norte de Fortaleza, de IDH elevado, é a região onde foram notificados os primeiros casos da doença, diferente da região periférica (região Sul), que apresenta baixo IDH, o que impacta diretamente no menor poder aquisitivo dessa população, dificultando a realização de viagens e turismo com destino aos países que possuíam casos confirmados da doença na época ${ }^{25}$.

Por conseguinte, evidenciou-se que em parcela expressiva dos bairros de Fortaleza, quanto maior o \% de ocupados $\geq 18$ anos, maior foi a incidência da doença. Este é um resultado esperado, dado que essa população tem maior dificuldade de manter o isolamento social devido suas características de emprego e renda, sendo aliado ao fato de que usam com mais frequência o transporte público, possuem mais moradores por domicílio, têm menor acesso a saneamento básico e saúde. Assim, têm mais chance de se infectar e disseminar a doença. Nesse cenário, a relação sujeito-social encontra-se fragilizada e, pior, a aparição desses sujeitos é renegada por patrões e Estado.

Dado o exposto, o Brasil é marcado por desigualdades e iniquidades no acesso e posse a bens, serviços e riquezas, fruto do trabalho coletivo acumulado por gerações, desigualmente distribuído ${ }^{4}$. Na saúde, as desigualdades geram diferentes possibilidades de usufruir dos avanços tecnológicos e divergem quanto às chances de exposição a fatores que determinam saúde, doença e morte ${ }^{3}$. Em vista disso, o número de casos e mortalidade vem crescendo na periferia das cidades de forma consubstancialmente acelerada e se interiorizando lentamente ${ }^{14}$. 
Além disso, enquanto o número de casos está se concentrando nas periferias, fala-se em relaxamento das medidas de isolamento. Entretanto, a quebra do isolamento e distanciamento social acarretou o aumento comprovado da transmissão da doença, acarretando elevando as taxas de hospitalização e número de casos graves ${ }^{5,6}$. Nesta seara, o acesso desigual a serviços de saúde impacta no desfecho clínico da doença, reafirmando a relevância de medidas de controle. Ademais, levam à reflexão da insuficiência de políticas públicas e descaso com os indicadores de vulnerabilidade social.

Este estudo mostra, principalmente, como a doença se manifestava no início da pandemia, quando, na maioria dos casos, eram testadas pessoas com renda mais elevada, dado que o diagnóstico à época se dava com base no teste molecular (RT-PCR), realizado em maior escala por pessoas com maior renda e acessibilidade a serviços de saúde.

Por esse motivo, observa-se a desigualdade nas taxas de subnotificação de COVID-19 nos diversos estados da federação, com os sete primeiros lugares ocupados por estados da região Norte e Nordeste. Logo, ampliar a testagem e diagnóstico da doença é um desafio que se impõe à sociedade brasileira e ao Sistema Único de Saúde ${ }^{2}$.

De maneira adicional, a relação entre pandemia e vulnerabilidade social também foi constatada em outros momentos históricos, como nas gripes espanhola, suína (H1N1) e SARS, constatando que as desigualdades sociais são determinantes para transmissão e para a severidade dessas doenças ${ }^{1}$.

Tendo como premissa que a vulnerabilidade em saúde se dá numa cena de aparição que é um espaço de reconhecimento pelo outro, é necessário refletir como a vida da periferia deve ser considerada num país com grande desigualdade social. Ainda, como reconhecer a sua vulnerabilidade? Nunca uma pergunta se fez tão pertinente e tão atual nessa pandemia que já ceifou a vida de milhares. Frente a problemática, para fundamentar políticas governamentais é vital analisar as repercussões da COVID-19 sobre os indivíduos em situação de vulnerabilidade, para que se possa reduzir a propagação da epidemia com ações direcionadas.

Por fim vale salientar que além de variáveis demográficas e espaciais de vulnerabilidade social à medida que a pandemia COVID-19 evolui, os países estão considerando políticas para proteger as pessoas com maior risco de doenças graves. Estes indivíduos tidos como aqueles com maior vulnerabilidade são portadores de doenças crônicas graves, idosos, sexo masculino, acometidos com doenças cardiovasculares e diabetes, estes fatores têm sido associados a risco aumentado de COVID-19 grave e morte ${ }^{26}$.

Fica claro que definir quem é vulnerável é complexo, e ultrapassa os fatores sociodemográficos e geográficos, por isso, devem ser considerados aqueles indivíduos em risco de doença grave, a evidência demostra que a proporção de pessoas com este tipo de vulnerabilidade pode constituir até 30\% da população em algumas regiões. Neste sentindo são essenciais esforços especiais para protegê-los, implementado estratégias multifacetadas direcionadas ao perfil da população ${ }^{27}$.

Este trabalho apresenta algumas limitações, como poucas referências anteriores que tenham ajudado a selecionar indicadores de vulnerabilidade social à COVID-19 e o fato de que os dados públicos disponíveis para análise no estudo podem sofrem impacto da subnotificação que se deve ao baixo índice de exames por milhão de habitantes. Além disso, há um atraso significativo na notificação dos resultados dos testes durante as primeiras semanas do surto de COVID-19. Ademais testou-se todos os casos suspeitos, bem como aqueles que estiveram em contato com um caso confirmado. No entanto, a baixa disponibilidade de testes de RT-PCR (reação em cadeia da polimerase de transcrição reversa) obrigou o Ministério da Saúde a recomendar o teste apenas para casos graves. Essa abordagem também foi estendida para aqueles em grupos de alto risco (por exemplo, profissionais de saúde).

Quanto aos indicadores de vulnerabilidade, é importante ressaltar que mesmo seguindo critérios do IBGE os dados utilizados são referentes ao censo de 2010, podendo ter sofrido modificações nos últimos 10 anos. Novos dados seriam coletados no ano de 2020 para uma produção mais acurada destes; entretanto, a mesma pandemia estudada impediu a realização de novo censo.

\section{Conclusão}

A influência dos indicadores de vulnerabilidade sobre a incidência evidenciou que, quanto maior a escolaridade, menor o risco para adoecimento por COVID-19, além de que a população em idade ativa para o trabalho é a que tem maior vulnerabilidade de exposição à infecção.

Assim, conhecer os indicadores de vulnerabilidade social no contexto pandêmico permite identificar e priorizar grupos com alta vulnera- 
bilidade, além de orientar e adaptar intervenções visando essa população. Urge a necessidade de remanejar recursos públicos e reforçar ações de promoção da saúde e medidas preventivas em locais de maior vulnerabilidade social para favorecer a formulação de novas políticas e programas de estabilização socioeconômica a essa clientela, diminuindo desigualdades sociais.

\section{Colaboradores}

VRF Cestari, RS Florêncio, GJB Sousa e TS Garces atuaram na concepção, análise e interpretação dos dados. RR Castro, LI Cordeiro e LLV Damasceno contribuíram substancialmente para a redação do artigo. TA Maranhão, VLMP Pessoa, MLD Pereira e TMM Moreira realizaram a supervisão e revisão crítica de todas as etapas do estudo. Todo os autores participaram da aprovação da versão final a ser publicada. 


\section{Referências}

1. Calmon TVL. As condições objetivas para o enfrentamento ao COVID-19: abismo social brasileiro, o racismo, e as perspectivas de desenvolvimento social como determinantes. NAU Social 2020; 11(20):131136.

2. Magno L, Rossi TA, Mendonça-Lima FW, Campos GB, Marques LM, Santos MP, Prado NMBL, Dourado I. Challenges and proposals for scaling up COVID-19 testing and diagnosis in Brazil. Cien Saude Colet 2020; 25(9):3355-3364.

3. Barreto ML. Desigualdades em saúde: uma perspectiva global. Cien Saude Colet 2017; 22(7):2097-2108.

4. Canuto R, Fanton M, Lira PIC. Social inequities in food consumption in Brazil: a critical review of the national surveys. Cien Saude Colet 2019; 24(9):3193212.

5. Moreira, RS. COVID-19: intensive care units, mechanical ventilators, and latent mortality profiles associated with case-fatality in Brazil. Cad Saúde Pública 2020; 36(5):e00080020.

6. Ribeiro F, Leist A. Who is going to pay the price of Covid-19? Reflections about an unequal Brazil. Int $J$ Equ Health 2020; 19:91-93.

7. Lima DLF, Dias AA, Rabelo RS, Cruz ID, Costa SC, Nigri FMN, Neri JR. Covid-19 in the State of Ceará: behaviors and beliefs in the arrival of the pandemic. Cien Saude Colet 2020; 25(5):1575-1586.

8. Sousa GJB, Garces TS, Cestari VRF, Florêncio RS, Moreira TMM, Pereira MLD. Mortality and survival of COVID-19. Epidemiol Infect 2020; 148:e123.

9. Fuck Júnior SCF. As condições desiguais de acesso à moradia em Fortaleza, Brasil. Rev Electr Geogr Cien Soc 2003; 7:146.

10. Barreto ML, Barros AJD, Carvalho MS, Codeço CTT, Halal PRC, Medronho RA, Struchiner CJ, Victora CG, Werneck GL. O que é urgente e necessário para subsidiar as políticas de enfrentamento da pandemia de COVID-19 no Brasil? Rev Bras Epidemiol 2020; 23:1-4.

11. The Lancet. Redefining vulnerability in the era of COVID-19. Lancet 2020; 294(10230):1089.

12. Santos JPC, Siqueira ASP, Praca HLF, Albuquerque HG. Vulnerabilidade a formas graves de COVID-19: uma análise intramunicipal na cidade do Rio de Janeiro, Brasil. Cad Saúde Pública 2020; 36(5):e00075720.

13. Rezende LFM, Thome B, Schweltzer MC, Souza-Júnior PRB, Szwarcwald CL. Adults at high-risk of severe cronavirus disease-2019 (Covid-19) in Brazil. Rev Saúde Pública 2020; 54:50.

14. Silva MHA, Procópio IM. A fragilidade do sistema de saúde brasileiro e a vulnerabilidade social diante da COVID-19. Rev Bras Promoc Saúde 2020; 33:10724.

15. Pires RRC. Os efeitos sobre grupos sociais e territórios vulnerabilizados das medidas de enfrentamento à crise sanitária da COVID-19: propostas para o aperfeiçoamento da ação pública. Brasília: IPEA;2020.

16. JPC, Siqueira ASP, Praça HLF, Albuquerque HG. Vulnerability to severe forms of COVID-19: an intra-municipal analysis in the city of Rio de Janeiro, Brazil. Cad Saúde Pública 2020; 36(5):e00075720.

17. CM, Silva IVM, Cidade NC. COVID-19 as a global disaster: challenges to risk governance and social vulnerability in Brazil. Amb Soc 2020; 23:1-12.
18. Florêncio RS, Moreira TM. Modelo de vulnerabilidade em saúde: esclarecimento conceitual na perspectiva do sujeito-social. Acta Paul Enferm 2021; 34(3): [No prelo].

19. Carmo ME, Guizardi FL. The concept of vulnerability and its meanings for public policies in health and social welfare. Cad Saúde Pública 2018; 34(3):e00101417.

20. Governo Estadual do Ceará. INTEGRASUS: Transparência da Saúde do Ceará [Internet]. Ceará: Governo Estadual do Ceará; 2020. Disponível em: https://integrasus.saude.ce.gov.br/

21. Tang Y, Wang S. Mathematic modeling of COVID-19 in the United States. Emerging Microbes Infections 2020; 9(1):827-829.

22. Mukherjee K. COVID-19 and Lockdown: Insights from Mumbai. Indian J Public Health 2020; 62(3):2018-2020.

23. Governo Estadual do Ceará. Secretaria de Vigilância em Saúde. Doença pelo novo coronavírus. Boletim Epidemiológico 2020; 447(3):1-18.

24. Chen Y, Leng K, Lu Y, Wen L, Qi Y, Gao W, Chen H, Bai L, An X, Sun B, Wang P, Dong J. Epidemiological features and time-series analysis of influenza incidence in urban and rural areas of Shenyang, China, 20102018. Epidemiol Infec 2020; 148:e29.

25. Lahiri A, Jha SS, Bhattacharya S, Soumalya R, Chakraborty A. Effectiveness of Preventive Measures against COVID-19: a Systematic Review of In Silico Modeling Studies in Indian Context. Indian J Public Health 2020; 62(3):2018-2020.

26. Clark A, Jit M, Warren-Gash C, Guthrie B, Wang HHX, Mercer SW, Sanderson C, McKee M, Troeger C, Ong KL, Checchi F, Perel P, Joseph S, Gibbs HP, Banerjee A, Eggo RM. Global, regional, and national estimates of the population at increased risk of severe COVID-19 due to underlying health conditions in 2020: a modelling study. Lancet 2020; 8(8): 003-1017.

27. Alwan NA, Burgess RA, Ashworth S, Beale R, Bhadelia, Bogaert D, Dowd J, Eckerle I, Goldman LR, Greenhalgh T, Gurdasani D, Hamdy A, Hanage WP, Hodcroft EB, Hyde Z, Kellam P, Kelly-Irving M, Krammer F, Lipsitch M, McNally A, McKee M, Pimenta AND, Priesemann V, Rutter H, Silver J, Sridhar D, Swanton C, Walensky RP, Yamey G, Ziauddeen H. Scientific consensus on the COVID-19 pandemic: we need to act now. Lancet 2020; 396(10260):E71-E72.

Artigo apresentado em 03/09/2020

Aprovado em 01/12/2020

Versão final apresentada em 03/12/2020

Editores-chefes: Romeu Gomes, Antônio Augusto Moura da Silva 
